\title{
Vehicular Slip Ratio Control Using Nonlinear Control Theory*
}

\author{
Yuichi IKEDA**, Takashi NAKAJIMA** and Yuichi CHIDA** \\ ** Shinshu University \\ 4-17-1 Wakasato, Nagano, Nagano 380-8553, Japan \\ E-mail: yikeda@shinshu-u.ac.jp
}

\begin{abstract}
In this paper, we discuss integrated vehicle slip ratio control under both deceleration and acceleration without the need for controller switching, and also propose a design method for such an integrated slip ratio controller based on the slip ratio dynamics. When a vehicle switches from acceleration to deceleration and vice versa, the slip ratio varies discontinuously. Here, the slip ratio is approximated to a continuous function by using a sigmoid function. And a controller is then designed by using feedback linearization based on the approximated slip ratio. The stability of the designed control system is proven by Lyapunov stability theorem. Furthermore, we propose a robust control method based on a disturbance observer and sliding mode control theory. Finally, the effectiveness of the proposed control method is verified through numerical simulation.
\end{abstract}

Key words : Vehicle, Slip Ratio, Feedback Linearization, Disturbance Observer, Sliding Mode Control

\section{Introduction}

In modern society, automobiles represent a fundamental means of transport. However, as the damage caused because of accidents is major, it is important to take measures to obviate the same. One of the reasons for such accidents includes vehicle spin or drift-out. A vehicle turns because of the yaw moment generated by the lateral forces exerted on the tire when steering. However, these lateral forces decrease rapidly when the tires slip or lock under rapid acceleration or deceleration, forcing the vehicle into a spin or drift-out. This occurs because a yaw moment sufficient to turn the vehicle cannot be generated. Therefore, most new vehicles are equipped with an anti-lock braking system (ABS) to prevent tire lock during deceleration, and a traction control system (TCS) to prevent tire spin during acceleration. Two separate systems are used because the relationship between the vehicle body velocity and the circumferential velocity of the tire is different in acceleration and deceleration, but both systems control the slip ratio of the vehicle.

A variety of slip ratio control methods have been proposed, such as sliding mode control $^{(1)-(3)}, \mathcal{H}_{\infty}$ control $^{(4)}$, and slip ratio estimator and observer ${ }^{(5),(6)}$. However, these control methods deal either with acceleration or with deceleration because the slip ratio varies discontinuously when the vehicle changes from acceleration to deceleration and vice versa. For this reason, two separate controllers are designed and switched when accelerating and decelerating. References (7) and (8) have been proposed a control method under both deceleration and acceleration without the need for controller switching based on difference or ratio of the wheel circumferential velocity and the vehicle body velocity, respectively. However, since these methods only utilize the static relationship of slip ratio, wheel circumferential velocity, and vehicle body velocity and do not consider the vehicle body dynamics, it is difficult to apply when slip ratio rapidly change such as rapid acceleration and/or deceleration for emergency avoidance. 


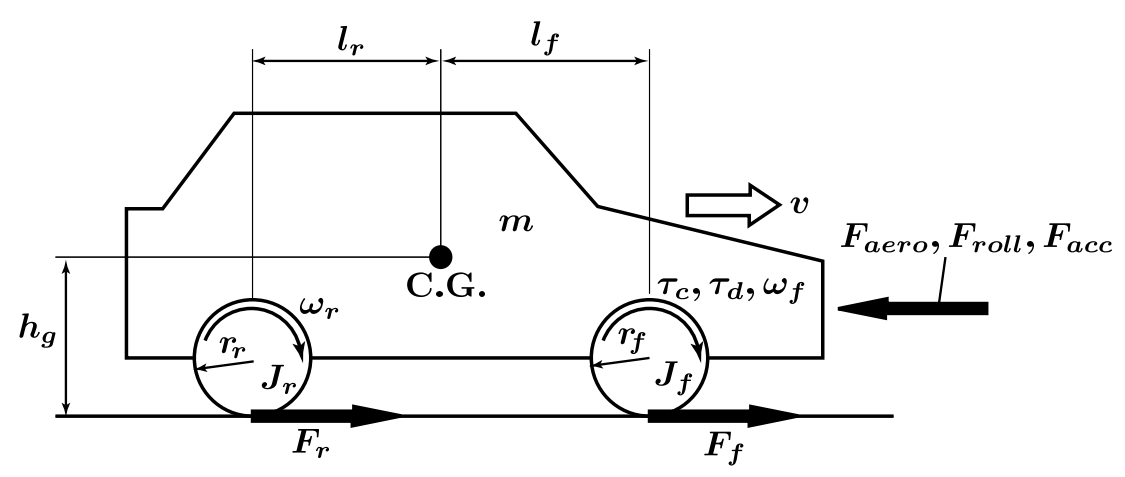

Fig. 1 Vehicle model

In this paper, we discuss integrates vehicle slip ratio control under both deceleration and acceleration without the need for controller switching based on the slip ratio dynamics and propose a design method for such a controller. The proposed method relies on feedback linearization based on a continuous approximation of the slip ratio using a sigmoid function. The stability of the control system is proven by using Lyapunov stability theorem. The effectiveness of the proposed method is further verified by numerical simulation.

\section{Vehicle Model}

The vehicle model considered in this paper is the front wheel drive vehicle, as shown in Fig. 1. The longitudinal motion of the vehicle and the rotational motion of the front and rear wheel can be described as follows:

$$
\begin{aligned}
& m \dot{v}=F_{f}+F_{r}-F_{d}, \\
& J_{f} \dot{\omega}_{f}=\tau_{c}+\tau_{d}-r_{f} F_{f}, \\
& J_{r} \dot{\omega}_{r}=-r_{r} F_{r}, \\
& F_{d}=F_{\text {aero }}+F_{\text {roll }}+F_{\text {acc }}, \\
& F_{\text {aero }}=\frac{\rho C_{d} A}{2} v^{2}, F_{\text {roll }}=C_{\text {roll }} m g, F_{\text {acc }}=\left(m+m_{r}\right) \dot{v},
\end{aligned}
$$

where $v[\mathrm{~m} / \mathrm{s}]$ denotes the vehicle body velocity, $\omega_{i}(i=f, r)[\mathrm{rad} / \mathrm{s}]$ is the angular velocity of each wheel, $\tau_{c}[\mathrm{Nm}]$ is the control torque, $\tau_{d}[\mathrm{Nm}]$ is the commanded torque from the driver, $F_{i}[\mathrm{~N}]$ is the driving/braking force on the each tire, $F_{\text {aero }}[\mathrm{N}]$ is the aerodrag force, $F_{\text {roll }}[\mathrm{N}]$ is the rolling resistance force, $F_{a c c}[\mathrm{~N}]$ is the acceleration resistance force, $m[\mathrm{~kg}]$ is the vehicle mass, $J_{i}\left[\mathrm{kgm}^{2}\right]$ is the inertial moment of the each wheel, $r_{i}[\mathrm{~m}]$ is the each wheel radius, $\rho$ $\left[\mathrm{kg} / \mathrm{m}^{3}\right]$ is the air density, $C_{d}[-]$ is the air resistance coefficient, $A\left[\mathrm{~m}^{2}\right]$ is the projected area of front of vehicle, $C_{\text {roll }}[-]$ is the rolling resistance coefficient, and $m_{r}[\mathrm{~kg}]$ is the equivalent inertia weight of rotative part of drive mechanism, respectively.

The slip ratio of each wheel $\lambda_{i}$ is expressed as follows considering that the driving force is applied when $r_{i} \omega_{i} \geq v$, and the braking force is applied when $r_{i} \omega_{i}<v$.

$$
\lambda_{i}=\frac{r_{i} \omega_{i}-v}{\max \left(r_{i} \omega_{i}, v\right)}
$$

The driving/braking force $F_{i}$ acting on the each tires is a function of the slip ratio $\lambda_{i}$, and the saturation characteristic changes according to the condition of road surface. In addition, it is well known that the driving/braking force has a maximum value when $\lambda_{i}$ is near \pm 0.2 . Since the vehicle dynamics in this paper considers the longitudinal direction only, the tire slip angle is considered zero and the considered tire force is the pure driving/braking force. In this paper, the Magic Formula tire model ${ }^{(9)}$, expressed by the following equation, is used to express these characteristics.

$$
F_{i}=\mu_{i} F_{z i} \sin \left\{C_{i} \tan ^{-1}\left(B_{i} \lambda_{i}\right)\right\},
$$




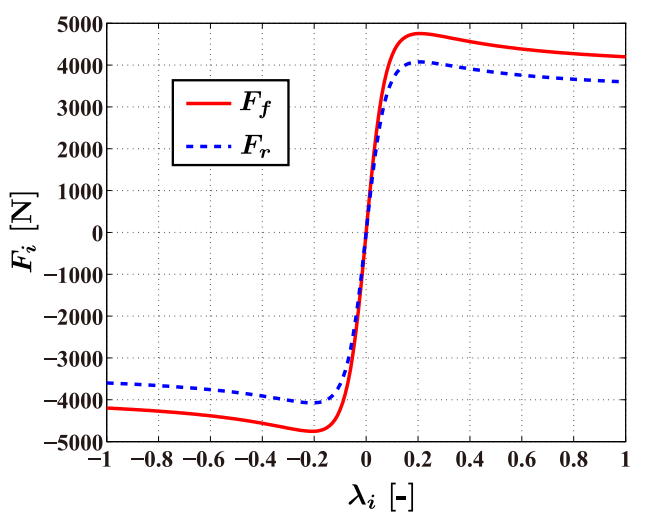

Fig. 2 Driving/braking force

where $\mu_{i}[-]$ is the friction coefficient of the road surface, $F_{z i}$ is the vertical load on each wheel, and $B_{i}$ and $C_{i}$ are the design parameters. By using Eq. (5) to express the driving/braking force $F_{i}$; the change in driving/breaking forces can be studied as shown in Fig. 2. The vertical load on each wheel $F_{z i}$ is expressed as follows using the longitudinal accelerations $\dot{v}$.

$$
\begin{aligned}
& F_{z f}=m_{s f} g+\frac{1}{2} \frac{l_{r}}{l} m_{s} g-m_{s} \frac{h_{g}}{l} \dot{v}, \\
& F_{z r}=m_{s r} g+\frac{1}{2} \frac{l_{f}}{l} m_{s} g+m_{s} \frac{h_{g}}{l} \dot{v},
\end{aligned}
$$

where $m_{s i}[\mathrm{~kg}]$ is the unspring weight of each wheel, $m_{s}[\mathrm{~kg}]$ is the spring mass of vehicle, $l_{i}$ $[\mathrm{m}]$ is the length from C.G. to each wheel, $l=l_{f}+l_{r}[\mathrm{~m}]$ is the wheelbase, $h_{g}[\mathrm{~m}]$ is the height from ground to C.G., and $g\left[\mathrm{~m} / \mathrm{s}^{2}\right]$ is the gravitational acceleration, respectively.

\section{Control System Design}

The slip ratio Eq. (4) varies discontinuously when the vehicle changes from acceleration to deceleration and vice versa. For this reason, separate controllers are used when accelerating and decelerating.

In this paper, in order to design an integrated controller for both acceleration and deceleration, the slip ratio $\lambda$ is approximated to a continuous function $\bar{\lambda}$ by using a sigmoid function. Then, based on this approximate slip ratio $\bar{\lambda}$, a controller which tracks the desired slip ratio $\lambda_{d}$ is designed by using feedback linearization. Finally, by using Lyapunov stability theorem, it is proven that $\lambda$ during acceleration and deceleration tracks $\lambda_{d}$ with the designed controller. Hereafter, we consider only control of front wheel slip ratio $\lambda_{f}$ and assume that all physical parameters are known, and that $v, \omega_{f}, \lambda_{f}, \tau_{d}, F_{f}, F_{r}, F_{\text {aero }}, F_{\text {roll }}$, and $F_{\text {acc }}$ are measurable.

\subsection{Approximation of slip ratio}

In this paper, the following sigmoid function is used:

$$
\sigma=\frac{1}{1+\exp \left\{-\gamma\left(\frac{r_{f} \omega_{f}}{v}-1\right)\right\}}
$$

where $\gamma$ is the design parameter. The approximate slip ratio $\bar{\lambda}_{f}$ is defined as follows:

$$
\bar{\lambda}_{f}=\frac{r_{f} \omega_{f}-v}{\sigma r_{f} \omega_{f}+(1-\sigma) v} .
$$

By setting $\gamma$ to a large value in Eq. (8), $\bar{\lambda}_{f}$ can approximate $\lambda_{f}$ with high accuracy. The time responses of $\lambda_{f}$ and $\bar{\lambda}_{f}$ are shown in Fig. 3, where a sine-wave torque with an amplitude of $700[\mathrm{~N}]$ and period of $5[\mathrm{~s}]$ is added as the commanded torque from the driver, $\tau_{d}$. In Fig. 3, $\gamma$ is set to a value of 40 . 


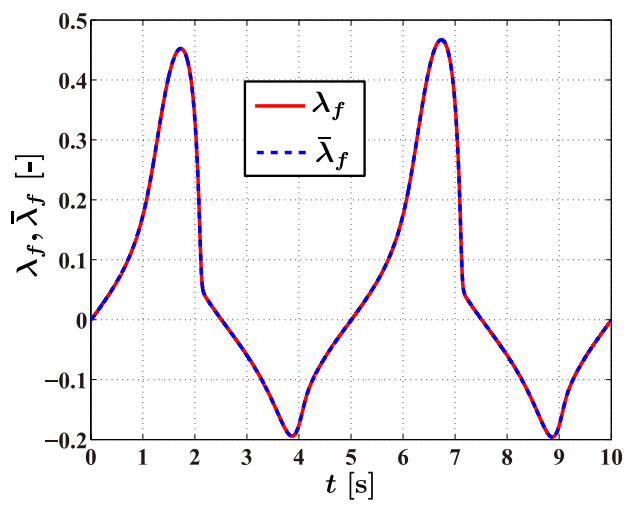

Fig. 3 Time response of $\lambda_{f}$ and $\bar{\lambda}_{f}$

\subsection{Controller design}

By differentiating Eq. (9) and substituting Eqs. (1) and (2), and $\dot{\sigma}$, the state equation with respect to $\bar{\lambda}_{f}$ is obtained as,

$$
\begin{aligned}
\dot{\bar{\lambda}}_{f}= & \frac{r_{f} v \dot{\omega}_{f}-r_{f} \omega_{f} \dot{v}-\dot{\sigma}\left(r_{f} \omega_{f}-v\right)^{2}}{\left\{\sigma r_{f} \omega_{f}+(1-\sigma) v\right\}^{2}} \\
= & \frac{r_{f}\left\{v^{2}(1+\Gamma)^{2}-\gamma \Gamma\left(r_{f} \omega_{f}-v\right)^{2}\right\}}{m J_{f} v^{2}\left(r_{f} \omega_{f}+\Gamma v\right)^{2}} \\
& \times\left\{m v\left(\tau_{c}+\tau_{d}-r_{f} F_{f}\right)-J_{f} \omega_{f}\left(F_{f}+F_{r}-F_{d}\right)\right\},
\end{aligned}
$$

where $\dot{\sigma}$ and $\Gamma$ are

$$
\begin{aligned}
& \dot{\sigma}=\frac{\gamma \Gamma\left\{m r_{f} v\left(\tau_{c}+\tau_{d}-r_{f} F_{f}\right)-J_{f} r_{f} \omega_{f}\left(F_{f}+F_{r}-F_{d}\right)\right\}}{m J_{f} v^{2}(1+\Gamma)^{2}}, \\
& \Gamma=\exp \left\{-\gamma\left(\frac{r_{f} \omega_{f}}{v}-1\right)\right\} .
\end{aligned}
$$

From Eq. (10), the control torque $\tau_{c}$ expressed by the following equation is obtained by using feedback linearization:

$$
\begin{aligned}
\tau_{c}=- & \frac{k J_{f} v\left(r_{f} \omega_{f}+\Gamma v\right)^{2}}{r_{f}\left\{v^{2}(1+\Gamma)^{2}-\gamma \Gamma\left(r_{f} \omega_{f}-v\right)^{2}\right\}} e_{\lambda_{f}} \\
& +\frac{\left(r_{f} m v+J_{f} \omega_{f}\right) F_{f}+J_{f} \omega_{f} F_{r}-J_{f} \omega_{f} F_{d}}{m v}-\tau_{d},
\end{aligned}
$$

where $k>0$ is the feedback gain; $e_{\lambda_{f}}=\bar{\lambda}_{f}-\lambda_{d}$ is the tracking error; and $\lambda_{d}$ is the desired slip ratio, which is determined as follows:

$$
\lambda_{d}=\left\{\begin{array}{cc}
a_{d}, & \text { if } \lambda_{f} \geq 0 \\
-a_{d}, & \text { if } \lambda_{f}<0
\end{array},\right.
$$

where $a_{d}$ is a positive constant value. In addition, the denominator of the first term in Eq. (11) is positive for all $v$ and $\omega_{f}$, that is,

$$
v^{2}(1+\Gamma)^{2}-\gamma \Gamma\left(r_{f} \omega_{f}-v\right)^{2}>0,{ }^{\vee} v, \omega_{f}
$$

by appropriately setting $\gamma$ of Eq. (8). Then, $e_{\bar{\lambda}_{f}}$ asymptotically converges to zero, that is, $\bar{\lambda}_{f}$ asymptotically converges to $\lambda_{d}$ because the error system becomes

$$
\dot{e}_{\bar{\lambda}_{f}}=\dot{\bar{\lambda}}_{f}=-k e_{\bar{\lambda}_{f}} \text {. }
$$


To control the actual slip ratio $\lambda_{f}$, we apply the following control law, in which the tracking error $e_{\lambda_{f}}$ of Eq. (11) is replaced by $e_{\lambda_{f}}=\lambda_{f}-\lambda_{d}$.

$$
\begin{aligned}
\tau_{c}=- & \frac{k J_{f} v\left(r_{f} \omega_{f}+\Gamma v\right)^{2}}{r_{f}\left\{v^{2}(1+\Gamma)^{2}-\gamma \Gamma\left(r_{f} \omega_{f}-v\right)^{2}\right\}} e_{\lambda_{f}} \\
& +\frac{\left(r_{f} m v+J_{f} \omega_{f}\right) F_{f}+J_{f} \omega_{f} F_{r}-J_{f} \omega_{f} F_{d}}{m v}-\tau_{d} .
\end{aligned}
$$

\section{Remark 1}

Design parameter $\gamma$ in Eq. (8) is the parameter to set the wave shape of sigmoid function $\sigma$. By setting $\gamma$ to a large value in Eq. (8), the wave shape of $\sigma$ comes close to step function that switches from 0 to 1 and vise versa in neighborhood of $\frac{r_{f} \omega_{f}}{v}=1$. However, when $\gamma$ is too large value, Eq. (13) does not satisfy. we confirm that the wave shape of $\sigma$ comes close to step function and Eq. (13) satisfies by setting $\gamma \geq 30$. In numerical simulation at $\$ 5$., $\gamma$ is set to a value of 40 .

\subsection{Stability of control system}

In this subsection, it is proven that $\lambda_{f}$ during acceleration and deceleration asymptotically converges to $\lambda_{d}$ under the control law Eq. (15). By differentiating Eq. (4) and substituting Eqs. (1), (2), and (15), the error equation with respect to $e_{\lambda_{f}}$ during acceleration and deceleration is obtained as follows:

- Acceleration $\left(r_{f} \omega_{f} \geq v\right)$ :

$$
\begin{aligned}
\dot{\lambda}_{f} & =\dot{e}_{\lambda_{f}} \\
& =\frac{v \dot{\omega}_{f}-\omega_{f} \dot{v}}{r_{f} \omega_{f}^{2}} \\
& =\frac{v}{J_{f} r_{f} \omega_{f}^{2}}\left(\tau_{c}+\tau_{d}-r_{f} F_{f}\right)-\frac{F_{f}+F_{r}-F_{d}}{m r_{f} \omega_{f}} \\
& =-\frac{k v^{2}\left(r_{f} \omega_{f}+\Gamma v\right)^{2}}{\left(r_{f} \omega_{f}\right)^{2}\left\{v^{2}(1+\Gamma)^{2}-\gamma \Gamma\left(r_{f} \omega_{f}-v\right)^{2}\right\}} e_{\lambda_{f}},
\end{aligned}
$$

- Deceleration $\left(r_{f} \omega_{f}<v\right)$ :

$$
\begin{aligned}
\dot{\lambda}_{f} & =\dot{e}_{\lambda_{f}} \\
& =\frac{r_{f}\left(v \dot{\omega}_{f}-\omega_{f} \dot{v}\right)}{v^{2}} \\
& =\frac{r_{f}}{J_{f} v}\left(\tau_{c}+\tau_{d}-r_{f} F_{f}\right)-\frac{r_{f} \omega_{f}\left(F_{f}+F_{r}-F_{d}\right)}{m v^{2}} \\
& =-\frac{k\left(r_{f} \omega_{f}+\Gamma v\right)^{2}}{v^{2}(1+\Gamma)^{2}-\gamma \Gamma\left(r_{f} \omega_{f}-v\right)^{2}} e_{\lambda_{f}} .
\end{aligned}
$$

We define the following candidate Lyapunov function:

$$
\mathcal{V}\left(e_{\lambda_{f}}\right)=\frac{1}{2} e_{\lambda_{f}}^{2}
$$

Then, the time derivative of Eq. (18) along the trajectories of the closed-loop system is as follows:

- Acceleration $\left(r_{f} \omega_{f} \geq v\right)$ :

$$
\dot{V}=-\frac{k v^{2}\left(r_{f} \omega_{f}+\Gamma v\right)^{2}}{\left(r_{f} \omega_{f}\right)^{2}\left\{v^{2}(1+\Gamma)^{2}-\gamma \Gamma\left(r_{f} \omega_{f}-v\right)^{2}\right\}} e_{\lambda_{f}}^{2},
$$




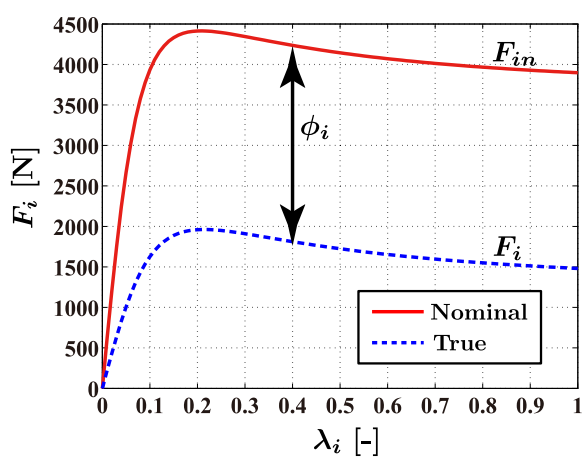

Fig. 4 Variations in the driving/braking force

Deceleration $(r \omega<v)$ :

$$
\dot{\mathcal{V}}=-\frac{k\left(r_{f} \omega_{f}+\Gamma v\right)^{2}}{v^{2}(1+\Gamma)^{2}-\gamma \Gamma\left(r_{f} \omega_{f}-v\right)^{2}} e_{\lambda_{f}}^{2}
$$

Obviously, $\dot{V}<0,{ }^{\forall} e_{\lambda_{f}} \neq 0$ holds for both acceleration and deceleration from Eq. (13). Since Lyapunov function $\mathcal{V}$ is positive definite and decrescent, $e_{\lambda_{f}}$ asymptotically converges to zero. That is, $\lambda_{f}$ during acceleration and deceleration asymptotically converges to $\lambda_{d}$.

\section{Robust Control System Design}

In the previous section, we assumed that all the physical parameters were known and the commanded torque from the driver $\tau_{d}$, the driving/braking force $F_{f}, F_{r}$, the aerodrag force $F_{\text {aero }}$, the rolling resistance force $F_{\text {roll }}$, and the acceleration resistance force $F_{\text {acc }}$ were measurable during controller design. However, in practice, the vehicle mass varies, for example, because of a varying number of passengers. Moreover, it is difficult to measure $\tau_{d}, F_{f}, F_{r}$, $F_{\text {aero }}, F_{\text {roll }}$, and $F_{\text {acc }}$ accurately. For actual vehicle control, a robust control system is required to account for physical parameter fluctuations and disturbances.

In this section, a robust control method based on a disturbance observer and the sliding mode control theory is proposed. Hereafter, we suppose that the physical parameter exhibiting fluctuation is vehicle mass $m$ and the inertial moment of the front wheel $J_{f}$, that other parameters are known, and that $\tau_{d}, F_{\text {aero }}, F_{\text {roll }}$, and $F_{\text {acc }}$ are unknown. Thus, $m$ and $J_{f}$ are expressed as the sum of the nominal value $m_{n}, J_{f n}$ and fluctuation $\Delta m, \Delta J_{f}$; that is, $m=m_{n}+\Delta m$, $J_{f}=J_{f n}+\Delta J_{f}$. Furthermore, by utilizing the true value $F_{i}(i=f, r)$ with the nominal value $F_{i n}$ and the bounded state-dependent coefficient $\phi_{i}$, the variation in the driving/braking force caused by changes in the road surface condition is expressed as follows (see Fig. 4):

$$
F_{i}=F_{\text {in }}+\phi_{i} .
$$

Thus, the longitudinal motion of the vehicle Eq. (1) and the rotational motion of the front wheel Eq. (2) can be described as follows:

$$
\begin{aligned}
& m_{n} \dot{v}=F_{f n}+F_{r n}+\Delta_{v}, \\
& J_{f n} \dot{\omega}_{f}=\tau_{c}-r_{f} F_{f n}+\bar{\tau}_{d},
\end{aligned}
$$

where are $\Delta m$ and $\bar{\tau}_{d}$ are

$$
\Delta_{v}=\phi_{f}+\phi_{r}-F_{d}-\Delta m \dot{v}, \bar{\tau}_{d}=\tau_{d}-r_{f} \phi_{f}-\Delta J_{f} \dot{\omega}_{f} .
$$

By differentiating Eq. (4) and substituting Eqs. (22) and (23), the state equation with respect to $\lambda_{f}$ during acceleration and deceleration is obtained as follows:

- Acceleration $\left(r_{f} \omega_{f} \geq v\right)$ :

$$
\dot{\lambda}_{f}=\dot{e}_{\lambda_{f}}=\frac{v}{J_{f n} r_{f} \omega_{f}^{2}}\left(\tau_{c}-r_{f} F_{f n}+\bar{\tau}_{d}\right)-\frac{F_{f n}+F_{r n}}{m_{n} r_{f} \omega_{f}}-\frac{\Delta_{v}}{m_{n} r_{f} \omega_{f}},
$$




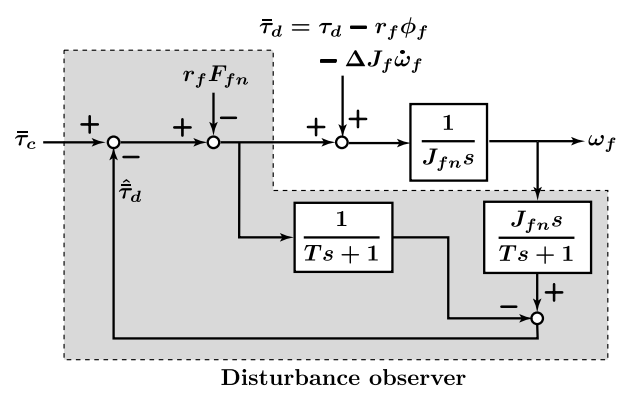

Fig. 5 Block diagram of disturbance observer

- Deceleration $(r \omega<v)$ :

$$
\dot{\lambda}_{f}=\dot{e}_{\lambda_{f}}=\frac{r_{f}}{J_{f n} v}\left(\tau_{c}-r_{f} F_{f n}+\bar{\tau}_{d}\right)-\frac{r_{f} \omega_{f}\left(F_{f n}+F_{r n}\right)}{m_{n} v^{2}}-\frac{r_{f} \omega_{f} \Delta_{v}}{m_{n} v^{2}},
$$

where $\bar{\tau}_{d}$ is estimated by the disturbance observer shown Fig. 5. The first order system in Fig. 5 is a low-pass filter that cuts off the high-frequency component. The estimated torque is represented by $\hat{\bar{\tau}}_{d}$.

To control the actual slip ratio $\lambda_{f}$, consider the following control law based on Eq. (15):

$$
\begin{aligned}
\tau_{c}= & \bar{\tau}_{c}-\hat{\bar{\tau}}_{d} \\
= & -\frac{k J_{f n} v\left(r_{f} \omega_{f}+\Gamma v\right)^{2}}{r_{f}\left\{v^{2}(1+\Gamma)^{2}-\gamma \Gamma\left(r_{f} \omega_{f}-v\right)^{2}\right\}} e_{\lambda_{f}}+\frac{\left(r_{f} m_{n} v+J_{f n} \omega_{f}\right) F_{f n}+J_{f n} \omega_{f} F_{r n}}{m_{n} v} \\
& -\beta \frac{e_{\lambda_{f}}}{\left|e_{\lambda_{f}}\right|}-\hat{\bar{\tau}}_{d}
\end{aligned}
$$

where $\beta>0$ is the design parameter. By substituting Eqs. (24) and (25) in Eq. (26), the state equation with respect to $e_{\lambda_{f}}$ under acceleration and deceleration is obtained as follows:

- Acceleration $\left(r_{f} \omega_{f} \geq v\right)$ :

$$
\begin{aligned}
\dot{e}_{\lambda_{f}}= & -\frac{k v^{2}\left(r_{f} \omega_{f}+\Gamma v\right)^{2}}{\left(r_{f} \omega_{f}\right)^{2}\left\{v^{2}(1+\Gamma)^{2}-\gamma \Gamma\left(r_{f} \omega_{f}-v\right)^{2}\right\}} e_{\lambda_{f}} \\
& -\frac{v}{J_{f n} r_{f} \omega_{f}^{2}}\left(\beta \frac{e_{\lambda_{f}}}{\left|e_{\lambda_{f}}\right|}+\frac{J_{f n} \omega_{f} \Delta_{v}}{m_{n} v}-\tilde{\bar{\tau}}_{d}\right),
\end{aligned}
$$

- Deceleration $\left(r_{f} \omega_{f}<v\right)$ :

$$
\dot{e}_{\lambda_{f}}=-\frac{k\left(r_{f} \omega_{f}+\Gamma v\right)^{2}}{v^{2}(1+\Gamma)^{2}-\gamma \Gamma\left(r_{f} \omega_{f}-v\right)^{2}} e_{\lambda_{f}}-\frac{r_{f}}{J_{f n} v}\left(\beta \frac{e_{\lambda_{f}}}{\left|e_{\lambda_{f}}\right|}+\frac{J_{f n} \omega_{f} \Delta_{v}}{m_{n} v}-\tilde{\bar{\tau}}_{d}\right),
$$

where $\tilde{\bar{\tau}}_{d}=\bar{\tau}_{d}-\hat{\bar{\tau}}_{d}$ is the estimate error. We define the following candidate Lyapunov function:

$$
\mathcal{V}\left(e_{\lambda_{f}}\right)=\frac{1}{2} e_{\lambda_{f}}^{2}
$$

Then, the time derivative of Eq. (29) along the trajectories of the closed-loop system is as follows:

- Acceleration $\left(r_{f} \omega_{f} \geq v\right)$ :

$$
\begin{aligned}
\dot{V}= & -\frac{k v^{2}\left(r_{f} \omega_{f}+\Gamma v\right)^{2}}{\left(r_{f} \omega_{f}\right)^{2}\left\{v^{2}(1+\Gamma)^{2}-\gamma \Gamma\left(r_{f} \omega_{f}-v\right)^{2}\right\}} e_{\lambda_{f}}^{2} \\
& -\frac{v}{J_{f n} r_{f} \omega_{f}^{2}} e_{\lambda_{f}}\left(\beta \frac{e_{\lambda_{f}}}{\left|e_{\lambda_{f}}\right|}+\frac{J_{f n} \omega_{f} \Delta_{v}}{m_{n} v}-\tilde{\bar{\tau}}_{d}\right),
\end{aligned}
$$


- Deceleration $\left(r_{f} \omega_{f}<v\right)$ :

$$
\dot{v}=-\frac{k\left(r_{f} \omega_{f}+\Gamma v\right)^{2}}{v^{2}(1+\Gamma)^{2}-\gamma \Gamma\left(r_{f} \omega_{f}-v\right)^{2}} e_{\lambda_{f}}^{2}-\frac{r_{f}}{J_{f n} v} e_{\lambda_{f}}\left(\beta \frac{e_{\lambda_{f}}}{\left|e_{\lambda_{f}}\right|}+\frac{J_{f n} \omega_{f} \Delta_{v}}{m_{n} v}-\tilde{\bar{\tau}}_{d}\right) .
$$

If the design parameter $\beta$ in Eqs. (30) and (31) satisfies

$$
\bar{\beta}=\beta-\left|\frac{J_{f n} \omega_{f} \Delta_{v}}{m_{n} v}-\tilde{\bar{\tau}}_{d}\right| \geq 0,
$$

then Eqs. (30) and (31) become

- Acceleration $\left(r_{f} \omega_{f} \geq v\right)$ :

$$
\begin{aligned}
\dot{V}= & -\frac{k v^{2}\left(r_{f} \omega_{f}+\Gamma v\right)^{2}}{\left(r_{f} \omega_{f}\right)^{2}\left\{v^{2}(1+\Gamma)^{2}-\gamma \Gamma\left(r_{f} \omega_{f}-v\right)^{2}\right\}} e_{\lambda_{f}}^{2}-\beta \frac{v}{J_{f n} r_{f} \omega_{f}^{2}}\left|e_{\lambda_{f}}\right| \\
& -\frac{v}{J_{f n} r_{f} \omega_{f}^{2}} e_{\lambda_{f}}\left(\frac{J_{f n} \omega_{f} \Delta_{v}}{m_{n} v}-\tilde{\bar{\tau}}_{d}\right) \\
\leq & -\frac{k v^{2}\left(r_{f} \omega_{f}+\Gamma v\right)^{2}}{\left(r_{f} \omega_{f}\right)^{2}\left\{v^{2}(1+\Gamma)^{2}-\gamma \Gamma\left(r_{f} \omega_{f}-v\right)^{2}\right\}} e_{\lambda_{f}}^{2}-\beta \frac{v}{J_{f n} r_{f} \omega_{f}^{2}}\left|e_{\lambda_{f}}\right| \\
& +\frac{v}{J_{f n} r_{f} \omega_{f}^{2}}\left|\frac{J_{f n} \omega_{f} \Delta_{v}}{m_{n} v}-\tilde{\bar{\tau}}_{d}\right|\left|e_{\lambda_{f}}\right| \\
= & -\frac{k v^{2}\left(r_{f} \omega_{f}+\Gamma v\right)^{2}}{\left(r_{f} \omega_{f}\right)^{2}\left\{v^{2}(1+\Gamma)^{2}-\gamma \Gamma\left(r_{f} \omega_{f}-v\right)^{2}\right\}} e_{\lambda_{f}}^{2} \\
& -\frac{v}{J_{f n} r_{f} \omega_{f}^{2}}\left(\beta-\left|\frac{J_{f n} \omega_{f} \Delta_{v}}{m_{n} v}-\tilde{\bar{\tau}}_{d}\right|\right)\left|e_{\lambda_{f}}\right| \\
= & -\frac{k v^{2}\left(r_{f} \omega_{f}+\Gamma v\right)^{2}}{\left(r_{f} \omega_{f}\right)^{2}\left\{v^{2}(1+\Gamma)^{2}-\gamma \Gamma\left(r_{f} \omega_{f}-v\right)^{2}\right\}} e_{\lambda_{f}}^{2}-\frac{\bar{\beta} v}{J_{f n} r_{f} \omega_{f}^{2}}\left|e_{\lambda_{f}}\right|,
\end{aligned}
$$

Deceleration $\left(r_{f} \omega_{f}<v\right)$ :

$$
\begin{aligned}
\dot{V} & =-\frac{k\left(r_{f} \omega_{f}+\Gamma v\right)^{2}}{v^{2}(1+\Gamma)^{2}-\gamma \Gamma\left(r_{f} \omega_{f}-v\right)^{2}} e_{\lambda_{f}}^{2}-\beta \frac{r_{f}}{J_{f n} v}\left|e_{\lambda_{f}}\right|-\frac{r_{f}}{J_{f n} v} e_{\lambda_{f}}\left(\frac{J_{f n} \omega_{f} \Delta_{v}}{m_{n} v}-\tilde{\bar{\tau}}_{d}\right) \\
& \leq-\frac{k\left(r_{f} \omega_{f}+\Gamma v\right)^{2}}{v^{2}(1+\Gamma)^{2}-\gamma \Gamma\left(r_{f} \omega_{f}-v\right)^{2}} e_{\lambda_{f}}^{2}-\beta \frac{r_{f}}{J_{f n} v}\left|e_{\lambda_{f}}\right|+\frac{r_{f}}{J_{f n} v}\left|\frac{J_{f n} \omega_{f} \Delta_{v}}{m_{n} v}-\tilde{\bar{\tau}}_{d}\right|\left|e_{\lambda_{f}}\right| \\
& =-\frac{k\left(r_{f} \omega_{f}+\Gamma v\right)^{2}}{v^{2}(1+\Gamma)^{2}-\gamma \Gamma\left(r_{f} \omega_{f}-v\right)^{2}} e_{\lambda_{f}}^{2}-\frac{r_{f}}{J_{f n} v}\left(\beta-\left|\frac{J_{f n} \omega_{f} \Delta_{v}}{m_{n} v}-\tilde{\bar{\tau}}_{d}\right|\right)\left|e_{\lambda_{f}}\right| \\
& =-\frac{k\left(r_{f} \omega_{f}+\Gamma v\right)^{2}}{v^{2}(1+\Gamma)^{2}-\gamma \Gamma\left(r_{f} \omega_{f}-v\right)^{2}} e_{\lambda_{f}}^{2}-\frac{\bar{\beta} r_{f}}{J_{f n} v}\left|e_{\lambda_{f}}\right| .
\end{aligned}
$$

Obviously, $\dot{V}<0,{ }^{\forall} e_{\lambda_{f}} \neq 0$ holds for both acceleration and deceleration from Eq. (13) and

$$
\frac{v}{J_{f n} r_{f} \omega_{f}^{2}}>0, \frac{r_{f}}{J_{f n} v}>0 .
$$

Since Lyapunov function $\mathcal{V}$ is positive definite and decrescent, $e_{\lambda_{f}}$ asymptotically converges to zero; that is, $\lambda_{f}$ during acceleration and deceleration asymptotically converges to $\lambda_{d}$. The block diagram of the proposed control system is shown in Fig. 6 


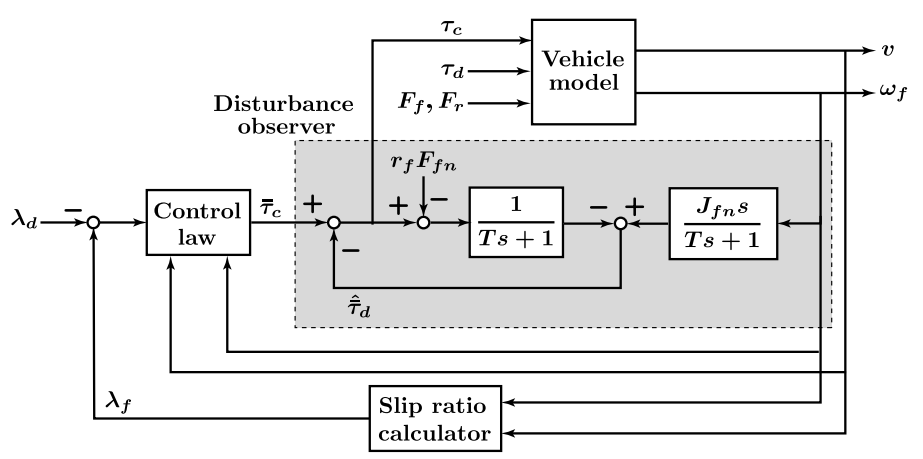

Fig. 6 Block diagram of control system

Table 1 Simulation parameters

\begin{tabular}{|c|c|c|c|c|c|}
\hline \multicolumn{7}{|c|}{ Vehicle parameters } \\
\hline$m_{n}$ & 1000 & $\Delta m$ & -200 & $J_{f n}$ & 0.869 \\
\hline$\Delta J_{f}$ & -0.174 & $J_{r}$ & 0.695 & $r_{f}, r_{r}$ & 0.311 \\
\hline$\rho$ & 1.241 & $C_{d}$ & 0.3 & $A$ & 2.5 \\
\hline$C_{r}$ & 0.01 & $m_{r}$ & 65 & $g$ & 9.8 \\
\hline$m_{s f}$ & 282.69 & $m_{s r}$ & 242.41 & $m_{s}$ & 950 \\
\hline$h_{g}$ & 0.6 & $l_{f}$ & 1.2 & $l_{r}$ & 1.4 \\
\hline \multicolumn{7}{|c|}{ Design parameters } \\
\hline$k$ & 4 & $\gamma$ & 40 & $\beta$ & 2000 \\
\hline$a_{d}$ & 0.2 & $T$ & 0.001 \\
\hline Magic Formula parameters (Dry asphalt) \\
\hline$B_{f}, B_{r}$ & 10.0 & $C_{f}, C_{r}$ & 1.4 & $\mu_{f}, \mu_{r}$ & 0.9 \\
\hline \multicolumn{7}{|c|}{ Magic Formula parameters (Wet asphalt) } \\
\hline$B_{f}, B_{r}$ & 7.0 & $C_{f}, C_{r}$ & 1.6 & $\mu_{f}, \mu_{r}$ & 0.4 \\
\hline \multicolumn{7}{|c|}{ Initial values } \\
\hline$v(0)$ & 25.0 & $\omega_{f}(0)$ & 80.5 & $\lambda_{f}(0)$ & 0 \\
\hline$\omega_{r}(0)$ & 80.5 & $\lambda_{r}(0)$ & 0 & \\
\hline
\end{tabular}

\section{Numerical Simulation}

Simulation was performed to verify the effectiveness of the proposed controller. The simulation parameters are listed in Table 1 . The commanded torque from driver $\tau_{d}$ was set as show in Fig. 7. In this simulation, assume that sign of $\tau_{d}$ is known. Moreover, the nominal values $F_{\text {in }}$ for the driving/braking force in the controller design were set for dry asphalt, whereas the true value $F_{i}$ was set as wet asphalt (see Fig. 8).

The results of numerical simulation are shown in Figs. 9 and 10. In the simulation, smoothing is implemented as,

$$
\frac{e_{\lambda_{f}}}{\left|e_{\lambda_{f}}\right|} \rightarrow \frac{e_{\lambda_{f}}}{\left|e_{\lambda_{f}}\right|+\varepsilon}, \varepsilon=0.05,
$$

to prevent chattering due to nonlinear inputs, the control torque $\tau_{c}$ was set to zero when $\tau_{d}=0$, and the desired slip ratio $\lambda_{d}$ was set to $a_{d}$ when $\tau_{d}>0$ and $-a_{d}$ when $\tau_{d}<0$.

Without the controller, the vehicle suffers tire locking during deceleration and the tire slip during acceleration. In addition, the vehicle velocity cannot be reduced or increased as adequate driving/braking force cannot be supplied when the tires lock or slip, respectively. By contrast, with the proposed controller, both tire lock and slip are prevented, despite physical parameter errors and variations in the road conditions. Thus, in this case, the vehicle can be decelerated and accelerated as the driving/braking force can reach the maximum value when the controller maintains the slip ratio within \pm 0.2 . Furthermore, although an estimate error exists due to the influence of a low-pass filter, $\bar{\tau}_{d}$ can be estimated by using the disturbance observer.

Then, in order to prove the effectiveness of the proposed controller, a comparison was made utilizing the wheel circumferential velocity tracking control method ${ }^{(8)}$. The block diagram of this control method is shown in Fig. 11. In Fig. 11, $K_{I}$ is the feedback gain of outer 


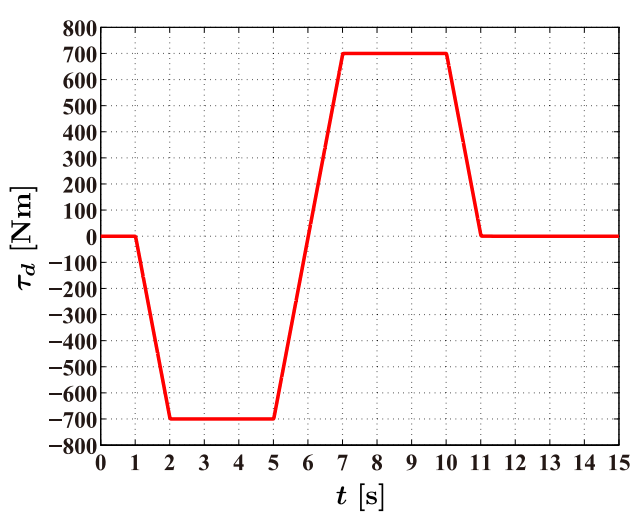

Fig. 7 Commanded torque from the driver $\tau_{d}$

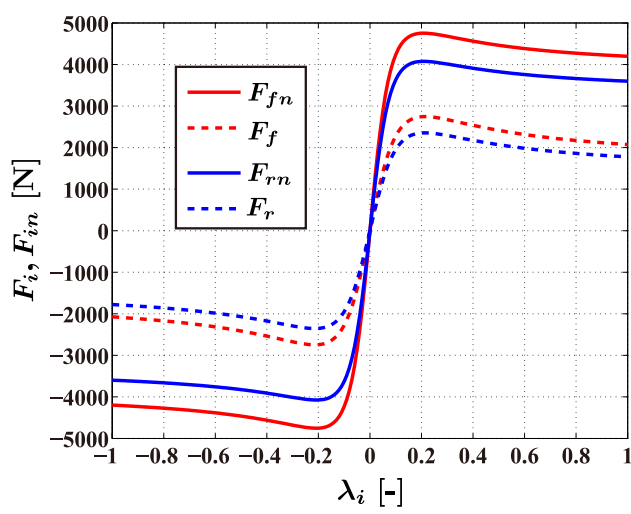

Fig. 8 Nominal values $F_{\text {in }}$ and true value $F_{i}$ at $\dot{v}=0$

loop, $C_{p}$ and $C_{I}$ are feedback gains of wheel seep controller (PI controller), $r_{f} \omega_{f}^{*}$ is the desired wheel circumferential velocity, which is determined as follows:

$$
r_{f} \omega_{f}^{*}=v+y v, y=\frac{r_{f} \omega_{f}}{v}-1 .
$$

In addition, the relation between slip ratio $\lambda_{f}$ and $y$ is expressed as follows:

$$
y=\left\{\begin{array}{ll}
\frac{\lambda_{f}}{1-\lambda_{f}}, & \text { if } r_{f} \omega_{f} \geq v \\
\lambda_{f}, & \text { if } r_{f} \omega_{f}<v
\end{array} .\right.
$$

By setting upper limit $y_{\max }$ and lower limit $y_{\min }$, and limiting the value of $y$ to $y_{\min } \leq y \leq y_{\max }$, the value of $\lambda_{f}$ can be limited within the range of $\lambda_{f, \min } \leq \lambda_{f} \leq \lambda_{f, \max } . K_{I}, C_{p}, C_{I}, y_{\max }$, and $y_{\min }$ are set as follows:

$$
K_{I}=0.02, C_{P}=500, C_{I}=1000, y_{\max }=0.25, y_{\min }=-0.2 .
$$

$y_{\max }$ and $y_{\min }$ are determined so as to set $\lambda_{f, \max }=0.2$ and $\lambda_{f, \min }=-0.2$. The results of numerical simulation using conventional method are shown in Fig. 12. Comparing proposed method with conventional one reveals that while the conventional method is prevented both tire lock and slip, the response of $\lambda_{f}$ get delayed for the commanded torque from the driver $\tau_{d}$. This result is attributed to the fact that the conventional method only utilize the static relationship of slip ratio and does not consider the vehicle body dynamics. Conversely, the response of $\lambda_{f}$ with proposed method reacts for $\tau_{d}$ without the delay. 


\section{Dynamics}

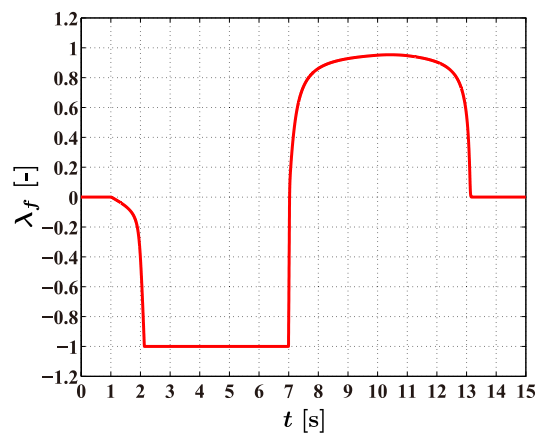

(a) $\lambda_{f}$

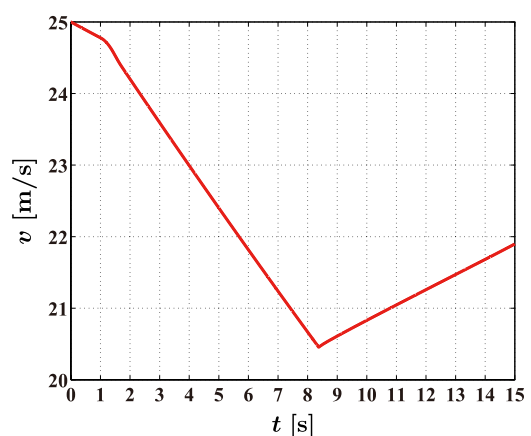

(b) $v$

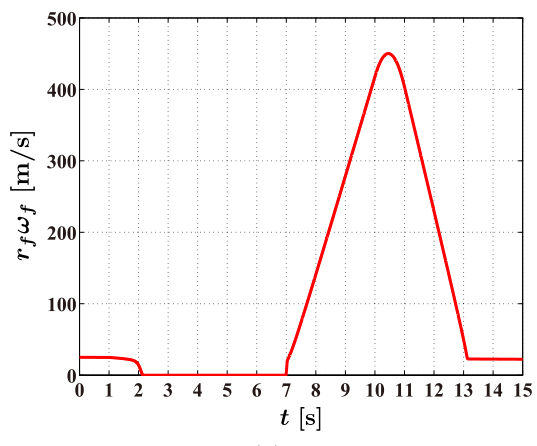

(c) $r_{f} \omega_{f}$

Fig. 9 Simulation results without control

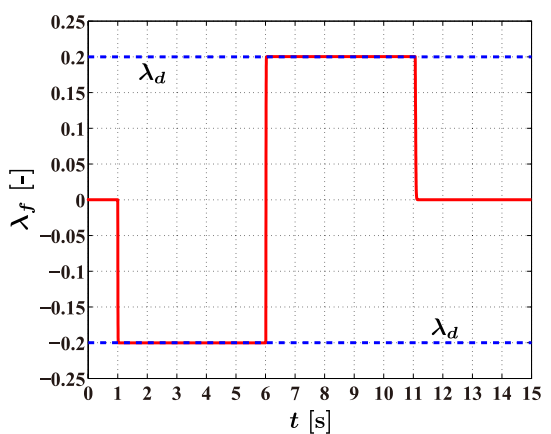

(a) $\lambda_{f}$

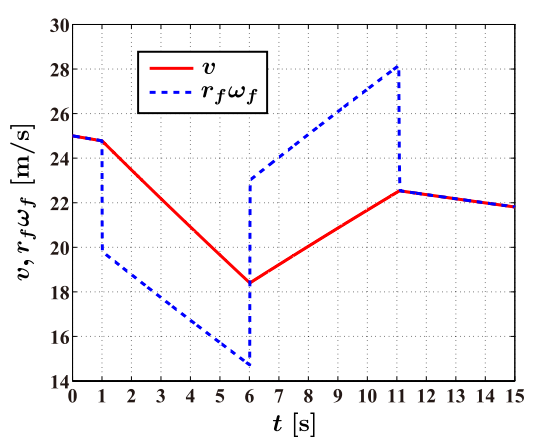

(b) $v$ and $r_{f} \omega_{f}$

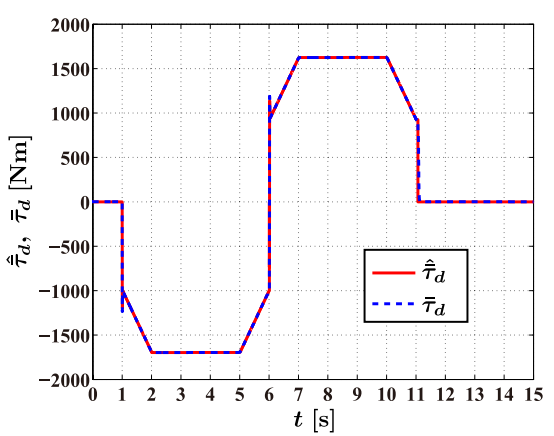

(c) $\hat{\bar{\tau}}_{d}$ and $\bar{\tau}_{d}$

Fig. 10 Simulation results with proposed controller. 


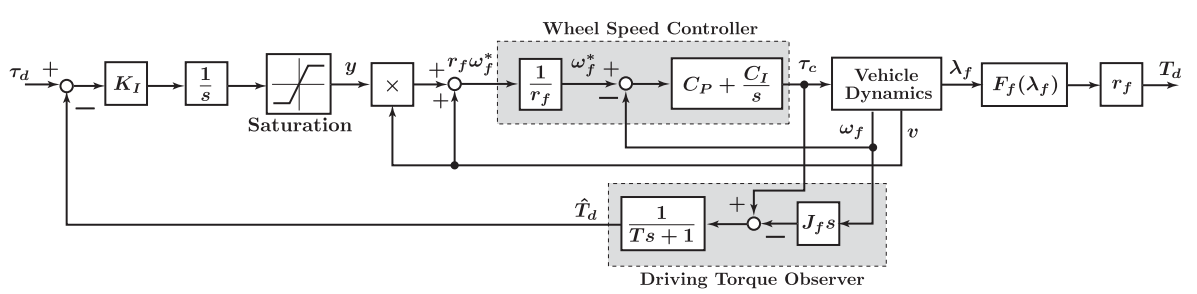

Fig. 11 Block diagram of conventional control method ${ }^{(8)}$

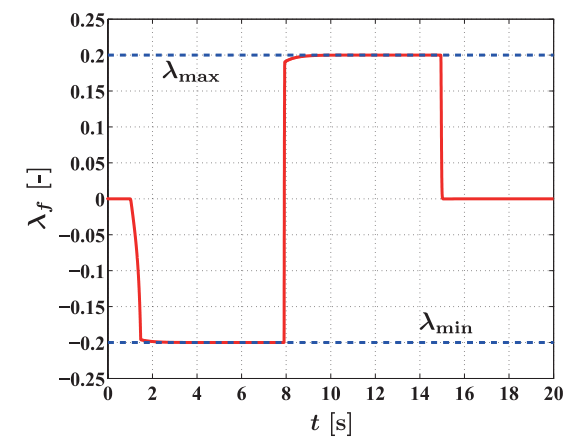

(a) $\lambda_{f}$

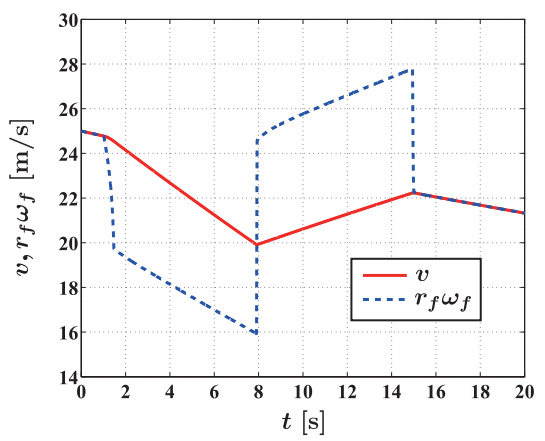

(b) $v$ and $r_{f} \omega_{f}$

Fig. 12 Simulation results with conventional $\operatorname{method}^{(8)}$

\section{Conclusion}

In this paper, we proposed an integrated control method based on the slip ratio dynamics for the vehicle slip ratio for both acceleration and deceleration. The proposed method relies on feedback linearization based on a continuous approximation of the slip ratio using a sigmoid function. The stability of the control system was proven by using Lyapunov stability theorem. Furthermore, in order to achieve robust stability against vehicle mass and wheel inertia fluctuation, variation in the driving/braking force caused by changes in the road surface conditions, and unknown commanded torque from the driver, aerodrag force and rolling resistance force, we proposed a robust control method based on a disturbance observer and sliding mode control theory. A numerical simulation verified the effectiveness of the proposed method.

\section{References}

( 1 ) Chikhi, F., El Hadri, A., and Cadiou, J.C., Optimal control for Anti-Braking System, Proceedings of the 2005 IEEE International Symposium on Intelligent Control (2005), pp.581-585.

( 2 ) Tanelli, M., Sartori, R., and Savaresi, S., Combining Slip and Deceleration Control for Brake-by-wire Control Systems: A Sliding-mode Approach, European Journal of Control, Vol.13, No.6 (2007), pp.593-611.

( 3 ) Kasahara, M., Kanai, Y., and Mori, Y., Vehicle Braking Control Using Sliding Mode Control- Switching Control for Speed and Slip Ratio -, ICROS-SICE International Joint Conference 2009 (2009), pp.4047-4052.

( 4 ) Lv, H., Jia, Y., Du, J. and Du, Q., ABS Composite Control Based on Optimal Slip Ratio, Proceedings of the 2007 American Control Conference (2007), pp.5748-5752.

( 5 ) Fujii, K., and Fujimoto, H., Traction Control based on Slip Ratio Estimation Without Detecting Vehicle Speed for Electric Vehicle, The Fourth Power Conversion Conference (2007), pp.688-693.

(6) Suzuki, T., and Fujimoto, H., Slip Ratio Estimation and Regenerative Brake Control without Detection of Vehicle Velocity and Acceleration for Electric Vehicle at Urgent Brake-turning, 2010 11th IEEE International Workshop on Advanced Motion Control (2010), pp.273-278. 
( 7 ) Shino, M., Ohshima, T., and Nagai, M., Wheel Velocity Control of Micro-Scale Electric Vehicle for Improving Direction Stability, Transactions of the Japan Society of Mechanical Engineers, Series C, Vol.70, No.694 (2004), pp.1680-1686 (in Japanese).

( 8 ) Yoshimura, M., and Fujimoto, H., Driving Torque Control Method for Electric Vehicle with In-Wheel Motors, IEEJ Transactions on Industry Applications, Vol.131-D, No.5 (2011), pp.721-728 (in Japanese).

( 9 ) Pacejka, H.B., and Besselink, I.J., Magic Formula Tyre Model with Transient Properties, Vehicle System Dynamics Supplement, Vol.27 (1997), pp.234-249. 